\title{
Visão, qualidade de vida e adesão medicamentosa em idosos com retinopatia diabética
}

\author{
Vision, quality of life and medication adherence in older adults with diabetic retinopathy \\ Visión, calidad de vida y adhesión a la terapia medicamentosa en los ancianos con \\ retinopatía diabética
}

Fernanda Freire Jannuzzi'; Fernanda Aparecida Cintra ${ }^{\text {II }}$; Maria Cecília Bueno Jayme GallanilI'; Andréa Mara Simões Torigoe ${ }^{I V}$

\begin{abstract}
RESUMO: O estudo objetivou analisar a influência da qualidade de vida relacionada à função visual (QVRFV) sobre a adesão medicamentosa e o efeito moderador da acuidade visual (AV) na relação QVRFV - adesão em idosos com retinopatia diabética. Trata-se de uma pesquisa transversal envolvendo idosos em uso de antidiabéticos orais/insulina e anti-hipertensivos. Avaliou-sea proporção de adesão e os cuidados no uso dos medicamentos. Utilizou-se o National Eyelnstitute Visual Function Questionnaire para avaliação da QVRFV. Empregou-se a análise de relação conjunta e a variância bivariada para testar o efeito moderador da AV na relação QVRFV - adesão. Dos 100 idosos avaliados, 58\% foram classificados como aderentes. Aqueles com pior QVRFV e baixa visão grave para longe mostraram 3.34 e 2.95 mais chance de não adesão, respectivamente. O efeito moderador estudado, entretanto, não foi observado. Os resultados ratificam que o efeito da AV e da QVRFV sobre a adesão ocorre de forma independente. Palavras-Chave: Adesão à medicação; qualidade de vida; retinopatia diabética; saúde do idoso.
\end{abstract}

\begin{abstract}
This cross-sectional study examined the influence of vision-related quality of life (VRQoL) on medication adherence, and the moderator effect of visual acuity (VA) in the relationship of VRQoL to adherence, in older adults with diabetic retinopathy. The study sample comprised elderly patients taking oral antidiabetic/insulin and antihypertensive drugs. Medication adherence rate, and its association with care taken in drug administration, were assessed. The National Eye Institute Visual Function Questionnaire was used to evaluate VRQoL. Analysis of the joint relationship and bivariate analysis of variance were applied to test the moderator effect of VA in the relationship between VRQoL and adherence. Of the 100 patients tested, 58\% classified as adherent. Those with worse VRQoL and severe visual impairment or blindness showed, respectively, 3.34 and 2.95 higher probability of non-adherence. However, the moderator effect studied was not observed. The data confirmed that the effects of VA and VRQoL on adherence were independent of each other.

Keywords: Medication adherence; quality of life; diabetic retinopathy; health of older adults.
\end{abstract}

RESUMEN: Este estudio tuvo como objetivo analizar la influencia de la calidad de vida relacionada con la función visual (CVRFV) sobre la adhesión a la terapéutica medicamentosa y el efecto moderador de la agudeza visual (AV) en relación CVRFV-adhesión en los ancianos con retinopatía diabética. Se trata de un estudio transversal involucrando a ancianos que usaban antidiabéticos orales/insulina y fármacos antihipertensivos. Se evaluó la proporción de la adhesión y los cuidados en cuanto al uso de medicamentos. Se utilizó el National Eye Institute Visual Function Questionnaire para evaluar CVRFV. Se utilizaron análisis de prueba conjunta y varianza bivariada para evaluar el efecto moderador de la AV en relación CVRFV-adhesión. De los 100 ancianos evaluados, un 58\% fue clasificado como adherentes. Los que obtuvieron peor CVRFV y discapacidad visual severa de distancia mostraron de 3.34 y 2.95 más posibilidad de no adhesión, respectivamente. No se observó, sin embargo, el efecto moderador estudiado. Estos datos confirman que el efecto de AV y CVRFV sobre la adhesión se produce de forma independiente.

Palabras Clave: Adhesión a la medicación; calidad de vida; retinopatía diabética; salud del anciano

\section{INTRODUÇÃO}

Entre as alterações oftalmológicas comumente manifestas no envelhecimento, algumas afecções destacam-se pela evolução progressiva, cronicidade e, especialmente, pela queda da visão, muitas vezes irreversível. A retinopatia diabética $(\mathrm{RD})$ constitui uma das mais prevalentes e incapacitantes complicações microangiopáticas que, com frequência, acomete os pacientes com Diabetes Mellitus (DM) ${ }^{1,2}$.

Os seguintes fatores de risco são apontados para a RD: tipo do DM, tempo de manifestação e controle

IEnfermeira, Doutoranda em Ciências da Saúde. Faculdade de Enfermagem, Universidade Estadual de Campinas. São Paulo, Brasil. E-mail: fernandafj@yahoo.com.br. "Enfermeira, Doutora em Enfermagem, Professor Associado, Faculdade de Enfermagem, Universidade Estadual de Campinas. São Paulo, Brasil. E-mail: fernanda@fcm.unicamp.br.

IIIEnfermeira, Doutora em Enfermagem, Professor Associado, Faculdade de Enfermagem - Universidade Estadual de Campinas. São Paulo, Brasil. Professor, Faculté dês SciencesInfirmières, UniverstiéLaval. Québec, Canadá. E-mail: ceciliag@fcm.unicamp.br.

IV Médica, Doutora em Ciências Médicas, Faculdade de Ciências Médicas, Universidade Estadual de Campinas. São Paulo, Brasil. E-mail: andreatorigoe@ hotmail.com. vArtigo extraído da Dissertação de Mestrado Qualidade de vida relacionada à função visual e adesão medicamentosa em idosos com retinopatia diabética apresentada ao Programa de Pós-Graduação em Enfermagem, Faculdade de Ciências Médicas, Universidade Estadual de Campinas. São Paulo, Brasil. 
metabólico da doença, níveis de hematócrito e hemoglobina, hemoglobina glicosilada, colesterol total, proteinúria e Hipertensão Arterial Sistêmica (HAS) ${ }^{3-5}$.

A maioria dos casos de cegueira em indivíduos adultos e idosos está relacionada à RD e pode ser evitada pelo emprego de medidas que inclui o diagnóstico precoce e a possibilidade de intervenção nos estágios iniciais da doença, bem como o controle da glicemia e da pressão arterial sistêmica, com hábitos de vida saudáveis e uso correto das medicações prescritas. Essas medidas retardam a progressão das alterações retinianas, sem reverter os danos já instalados ${ }^{6}$.

As alterações visuais decorrentes da RD têm impacto significativo na qualidade de vida. $O$ processo de percepção dos sinais e sintomas é vivenciado de forma única, pessoal, e requer atitude de superação e enfrentamento frente às perdas?

Nessa perspectiva, a identificação dos fatores relacionados à adesão medicamentosa em idosos portadores de RD possibilita o planejamento de estratégias de ação para o controle da doença, melhora do prognóstico visual e, por conseguinte, prevenção de incapacidades que interferem na qualidade de vida relacionada à saúde (QVRS) destes sujeitos.

Baron e Kenny ${ }^{8}$ apresentam um modelo de efeito moderador entre variáveis de interesse, no qual definem variável moderadora como aquela que afeta a direção e/ou a força da relação entre a variável preditora ou independente e a variável critério ou dependente. Ver Figura 1.

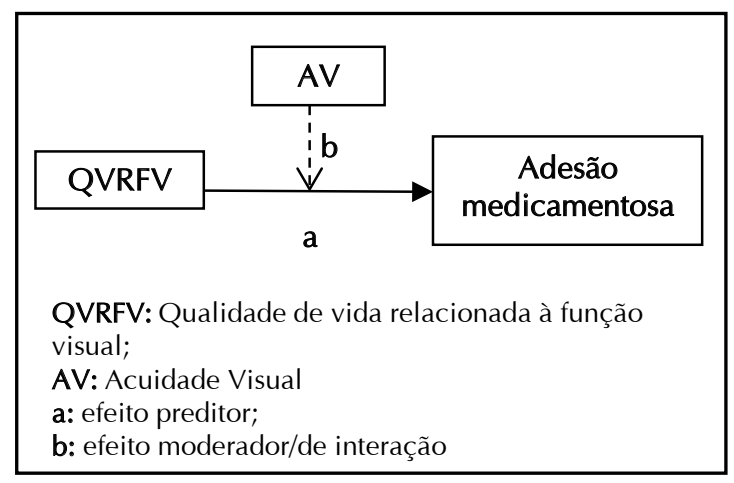

FIGURA 1: Modelo hipotético de relação entre as variáveis.

Com base nesse modelo, assume-se o pressuposto de a QVRFV é preditora da adesão medicamentosa, cuja associação é moderada pela acuidade visual (AV).

Assim, o presente estudo ${ }^{V}$ teve como objetivo analisar a influência da QVRFV sobre a adesão medicamentosa, bem como o efeito moderador da AV na relação QVRFV e adesão em idosos com retinopatia diabética.

\section{REVisão DE Literatura}

O conceito de adesão medicamentosa pode ser compreendido como a utilização dos medicamentos prescritos em pelo menos $80 \%$ do seu total, observando horários, doses, tempo de tratamento, e representa, assim, a etapa final do que se sugere como uso racional de medicamentos ${ }^{9}$.

Para os idosos, que comumente apresentam associação de comorbidades e são sujeitos ao multiuso de fármacos, a não adesão à terapêutica medicamentosa pode representar risco para a saúde, pelo controle inadequado das doenças. Numa perspectiva econômica, a não adesão também pode implicar maior utilização dos serviços de saúde, gastos adicionais em hospitalização e uso de uma terapia medicamentosa ainda mais extensa ${ }^{10,11}$.

O uso de medicamentos pelos idosos sofre influência de fatores relacionados à idade, como visão, audição e memória, e outros que incluem suporte ambiental, social e financeiro, além de necessidades percebidas ${ }^{12}$. No entanto, não há evidências na literatura sobre a associação, ou o efeito, da AV e da QVRS, ou mais especificamente relacionada à função visual, sobre a adesão medicamentosa em idosos com retinopatia.

\section{Metodologia}

Pesquisa transversal descritiva/exploratória, do tipo correlacional, realizada no Ambulatório de Oftalmologia de um hospital universitário no interior do Estado de São Paulo.

A amostra foi constituída por 100 idosos com diagnóstico médico de RD, em uso contínuo de medicamentos anti-hipertensivos e/ou antidiabéticos orais/insulina, acompanhados no ambulatório selecionado. Foram excluídos os sujeitos portadores de outra afecção ocular responsável pela baixa visão e aqueles submetidos a cirurgias oculares no período inferior a 30 dias da coleta de dados ${ }^{13}$.

O tamanho da amostra foi estimado considerandose a natureza da pesquisa e a extensão dos instrumentos de coleta de dados. A partir das variáveis preditoras (AV para perto e para longe, associação das duas medidas da AV e QVRFV), determinou-se o valor de oito sujeitos por variável com acréscimo de 50 sujeitos a esse valor ${ }^{14}$.

A coleta de dados foi realizada no período entre fevereiro e dezembro de 2008, por meio de entrevista individual estruturada.

$\mathrm{Na}$ véspera da coleta de dados, era solicitado por contato telefônico aos pacientes, potencialmente elegíveis para a pesquisa, que trouxessem as receitas médicas dos medicamentos em uso contínuo, acompanhadas dos frascos ou cartelas das respectivas drágeas/ comprimidos. Esta medida visava garantir a identificação de todos os medicamentos prescritos, bem como a forma indicada no receituário para a sua utilização ${ }^{15}$. 
Caracterização dos sujeitos: questionário que contempla o perfil sociodemográfico (idade, gênero, escolaridade e renda familiar), caracterização clínica autor relatada e medida da AV para longe (Tabela Optométrica de Snellen) e para perto (Tabela de Jaeger), com correção óptica se os pacientes fizessem uso. Os valores desta medida foram obtidos dos registros nos prontuários dos pacientes, após a avaliação oftalmológica realizada na data da entrevista.

Os idosos foram agrupados conforme a AV para longe (Grupos I, II, III e IV), segundo a Organização Mundial da Saúde ${ }^{16}$. Na classificação da AV para perto foram criados os grupos $\mathrm{A}, \mathrm{B}$ e $\mathrm{C}^{17,18}$. Estas classificações sofreram pequenas adaptações para esta pesquisa. Além destes agrupamentos, criou-se outros dois grupos (1 e 2) com a combinação de ambas as distâncias, sendo o grupo 1 considerado com visão normal ou próxima do normal para longe e para perto ( $\mathrm{AV} \geq 20 / 60$ e $\geq \mathrm{J} 3$ ) e grupo 2 com baixa visão para longe e perto $(\mathrm{AV}<20 / 60 \mathrm{e} / \mathrm{ou}<\mathrm{J} 3)$.

Foi utilizado o National Eye Institute Vision Function Questionnaire (NEI VFQ-25), instrumento cuja finalidade consiste em medir a influência da visão nas múltiplas dimensões da QVRS, bem como o bem-estar emocional e o funcionamento social. Trata-se em um questionário específico para ser aplicado em diversas condições oculares crônicas ${ }^{19}$. A adaptação cultural no Brasil foi realizada por Fonseca ${ }^{20}$. O NEI VFQ-25 é composto por 25 itens reunidos em 12 domínios ou subescalas. $\mathrm{O}$ escore total e de cada domínio do instrumento varia numa escala de zero a 100 , sendo zero a pior e 100 a melhor qualidade de vida.

A medida da adesão medicamentosa aos medicamentos antidiabéticos orais/insulina e anti-hipertensivos foi considerada segundo dois critérios: proporção de adesão e avaliação global da adesão.

Proporção de adesão: avaliada por meio de instrumento composto por quatro quadros que compreendem: 1. Descrição do nome, dosagem e posologia de todos os fármacos prescritos; 2. Descrição da forma de utilização de cada fármaco prescrito, segundo dose e posologia, nas 24 horas anteriores à entrevista; 3. Descrição da utilização dos mesmos na semana anterior à entrevista; e, 4. Descrição da utilização no mês anterior. Os quadros 2 e 3 , referentes ao uso dos fármacos na véspera e semana anterior, respectivamente, visam obter respostas mais acuradas por meio da minimização do viés da memória ${ }^{21}$. A adesão foi calculada a partir das doses omitidas no mês anterior, declaradas pelo próprio paciente, mediante o seguinte cálculo: [(doses prescritas - doses perdidas) x $100 /$ doses prescritas $]^{22}$. Para efeito de cálculo, os sujeitos que faziam uso da dose superior à prescrita tiveram seus valores de adesão convertidos para os índices correspondentes, inferiores a 100\%, proporcionalmente (uma vez que o uso da medicação além do prescrito implica em não adesão) ${ }^{23}$. Para os sujeitos que faziam uso de mais de um medicamento, a proporção final da adesão foi calculada pela média das porcentagens de adesão de cada fármaco. A proporção de adesão foi tratada como variável contínua (considerando-se a média da proporção do uso dos medicamentos), e como variável categórica: dose adequada e dose insuficiente, tendo como ponto de corte o valor de $80 \%$ de adesão.

Avaliação global da adesão: além da proporção do uso dos medicamentos também foi avaliada a sua posologia, ou seja, o número de medicamentos ingeridos e sua associação com marcadores temporais: jejum, café da manhã, almoço, jantar. Assim, para avaliação global da adesão, os pacientes foram classificados em quatro grupos: I - Dose e cuidados adequados à prescrição; II - Dose adequada e cuidados inadequados; III - Dose insuficiente e cuidados adequados; e, IV - Dose e cuidados inadequados.

Os dados foram inicialmente inseridos no programa Statistical Package for the Social Sciences (15.0 for Windows) para as análises: descritiva, de comparação (teste Qui-Quadrado ou Exato de Fisher, MannWhitney, Kruskal-Wallis e coeficiente de correlação de Spearman) e de relação conjunta (análise de regressão logística e modelo univariado). Análise de variância bivariada (QVRFV x AV) foi realizada para testar o efeito moderador da AV na relação QVRFV - adesão.

O nível de significância adotado para os testes estatísticos foi $5 \%$.

O estudo foi aprovado pelo Comitê de Ética em Pesquisa local (Parecer no 777/2007). Todos os sujeitos arrolados assinaram o Termo de Consentimento Livre e Esclarecido, após esclarecimento sobre a garantia do anonimato e a liberdade para desistência em qualquer momento.

\section{Resultados}

Os sujeitos apresentaram média de idade de $69,5( \pm 7,1)$ anos; a maioria era formada por mulheres (62\%). A escolaridade foi baixa, com média de 4,0 anos de estudo $( \pm 3,0)$. Todos tinham diagnóstico médico de DM e 85\% de HAS. Do total de fármacos em uso contínuo pelos idosos, em média 3,3 $( \pm 1,5)$ eram destinados ao tratamento de HAS e/ou DM.

Considerando a estratificação da AV para longe, os idosos apresentaram a seguinte distribuição: 44\% visão normal ou próxima do normal; 37\% baixa visão moderada; $17 \%$ baixa visão grave/profunda; e 2\% visão próxima a cegueira ou cegueira.Em relação à estratificação da visão para perto, 63\% dos idosos pertenceram ao grupo com visão normal ou próxima do normal; $15 \%$ estavam no grupo de baixa visão; e $22 \%$ apresentavam cegueira. Considerando a associação das duas distâncias, para longe e para perto da AV, a maior porcentagem (62\%) concentrou-se no grupo com baixa visão. 
Na aplicação do NEI VFQ-25, as maiores pontuações foram observadas nos domínios Visão de Cores, Dirigir Automóvel e Função Social, o que revela uma tendência à melhor QVRFV. Os escores mais baixos foram obtidos nas subescalas Saúde Geral e Visão Geral. Apenas 10 sujeitos responderam aos itens incluídos no domínio Dirigir Automóvel. Foi observado escore total médio do instrumento igual a 71,8 $( \pm 22,7)$, com variação entre 12 e 99,4.

A análise de comparação entre a AV (para longe, para perto e ambas) e o escore total do instrumento NEI VFQ-25 mostrou que os indivíduos com pior visão tiveram escores mais baixos no instrumento de avaliação da QVRFV. Ou seja, a pontuação obtida no NEI VFQ-25 teve diferença significativa entre os grupos I, II, III e IV da AV para longe (p-valor $<0,001$ ), entre os grupos $\mathrm{A}, \mathrm{B} \mathrm{e} \mathrm{C}$ da $\mathrm{AV}$ para perto ( $\mathrm{p}$-valor $<$ 0,001 ), bem como nos grupos 1 e 2 na combinação de AV para perto e longe $(\mathrm{p}$-valor $<0,001)$.
Entre os idosos avaliados, $85 \%$ utilizavam medicamentos anti-hipertensivos. Todos faziam uso de medicações para o tratamento do diabetes, sendo que $48 \%$ eram insulinodependentes.

No mês que antecedeu a entrevista, os sujeitos relataram fazer uso de $86,3 \%( \pm 18,0)$ das doses prescritas de medicamentos para HAS e/ou DM. Pouco mais da metade do grupo estudado (58\%) referiu utilizar $80 \%$ ou mais da dose prescrita e seguir os cuidados necessários na tomada dos medicamentos (Grupo I). Os demais idosos (Grupos II, III e IV) foram agregados em uma única classe. Assim, constituíram-se duas classes: Aderentes $(\mathrm{n}=58)$, formada pelos sujeitos do Grupo I e Não-aderentes ( $\mathrm{n}=42$ ), com os indivíduos dos demais grupos. Ver Tabela 1.

Para identificar a possível associação entre as variáveis independentes (AV e QVRFV) e a não adesão à terapêutica medicamentosa, foi realizada uma análise exploratória testando a correlação entre as variáveis, conforme mostra a Tabela 2.

TABELA 1: Adesão medicamentosa segundo os critérios da proporção da adesão e classificação de acordo com a adequação da dose e cuidados. Hospital Universitário, Estado de São Paulo, 2008.

\begin{tabular}{|c|c|c|c|}
\hline \multicolumn{4}{|c|}{ Proporção da adesão medicamentosa } \\
\hline \multirow{2}{*}{ Medicamentos } & Total & Indivíduos diabéticos & $\begin{array}{l}\text { Indivíduos diabéticos e } \\
\text { hipertensos }\end{array}$ \\
\hline & $(n=100)$ & $(n=15)$ & $(n=85)$ \\
\hline Antidiabéticos orais/insulina & $87,5( \pm 19,5)$ & $86,7( \pm 13,3)$ & $87,6( \pm 20,5)$ \\
\hline Anti-hipertensivos & $86,2( \pm 21,1)$ & --- & $86,2( \pm 21,1)$ \\
\hline $\begin{array}{l}\text { Antidiabéticos orais/insulina e } \\
\text { anti-hipertensivos }\end{array}$ & $86,3( \pm 18,0)$ & --- & $86,3( \pm 18,7)$ \\
\hline \multicolumn{3}{|c|}{ Classificação da avaliação global à terapêutica medicamentosa $(n=100)$} & $\%$ \\
\hline \multicolumn{4}{|l|}{ Aderentes } \\
\hline \multicolumn{3}{|c|}{ Doses e cuidados adequados à prescrição } & 58 \\
\hline \multicolumn{4}{|l|}{ Não-aderentes } \\
\hline \multicolumn{3}{|c|}{ Dose correta e cuidados inadequados } & 15 \\
\hline \multicolumn{3}{|c|}{ Dose insuficiente e cuidados adequados } & 11 \\
\hline Dose e cuidados inadequados & & & \\
\hline
\end{tabular}

TABELA 2: Análise de regressão logística univariada para a não-adesão medicamentosa (N=100).

\begin{tabular}{llccc}
\hline \multicolumn{1}{c}{ Variáveis } & \multicolumn{1}{c}{ Categorias } & p-valor & O.R.(*) & IC 95\%(**) \\
\hline \multirow{3}{*}{ AV para longe } & Normal (ref.) & -- & 1.00 & -- \\
& Baixa visão moderada & 0.195 & 1.82 & $0.74-4.51$ \\
& Baixa visão grave ou cegueira & 0.056 & 2.95 & $0.97-8.94$ \\
\hline \multirow{2}{*}{ AV para perto } & Normal (J1, J2 e J3) (ref.) & -- & 1.00 & -- \\
& Baixa Visão (J4, J5 e J6) & 0.802 & 1.16 & $0.37-3.67$ \\
& Cegueira (<J6) & 0.069 & 2.51 & $0.93-6.78$ \\
\hline \multirow{2}{*}{ AV para longe e perto } & Normal (ref.) & -- & 1.00 & - \\
& Baixa visão ou cegueira & 0.101 & 2.03 & $0.87-4.73$ \\
\hline \multirow{2}{*}{ NEl VFQ-25 } & $75-100$ (ref.) & -- & 1.00 & - \\
(categorizado) & $50-74$ & 0.269 & 1.74 & $0.65-4.64$ \\
& $0-49$ & 0.024 & 3.34 & $1.17-9.50$ \\
\hline
\end{tabular}

(*) O.R. (Odds Ratio) = Razão de risco para não-adesão medicamentosa $(\mathrm{n}=42$ Não-aderente e $\mathrm{n}=58 \mathrm{Aderente})$. (**)IC $95 \%=$ Intervalo de $95 \%$ de confiança para a razão de risco.

Ref.: categoria utilizada como referência para a análise.

AV: acuidade visual. 
A análise aponta que os sujeitos com pior QVRFV (escore total 0-49 no NEI VFQ-25) e aqueles com baixa visão grave ou cegueira para longe têm $3.34(\mathrm{p}=0.024)$ e $2.95(p=0.056)$ mais chance de não adesão à terapêutica medicamentosa, quando comparados aos sujeitos com melhor QVRFV e AV para longe, respectivamente.

Para estudar o efeito moderador da AV (para longe, para perto e ambas) na relação entre QVRFV e não adesão medicamentosa foi empregado o procedimento descrito por Cohen \& Cohen em 1983, adicionando o produto (termo de interação) das variáveis independente e moderadora na equação de regressão. Considerando X (QVRFV) como variável independente, Z (AV) como variável moderadora e $Y$ (não adesão) como variável dependente, foi feita a regressão de Y em X, Z e XZ. O efeito moderador é indicado pelo efeito significante de $\mathrm{XZ}$, enquanto $\mathrm{X}$ e Z são controlados ${ }^{8}$. O modelo empregado, entretanto, não confirmou o efeito moderador da AV na relação entre a QVRFV e a adesão.

\section{Discussão}

$\mathrm{Na}$ amostra estudada, a maior parte dos sujeitos concentrou-se no grupo com baixa visão, na associação das medidas da AV para longe e para perto. A literatura aponta que em qualquer estágio da $\mathrm{RD}$ pode ocorrer diminuição importante da $\mathrm{AV}$, causada pelo edema macular, embora seja mais freqüente nas fases avançadas ${ }^{7,24}$.

Destacam-se, ainda, os escores encontrados no instrumento NEI VFQ-25. As médias mais elevadas, e que retratam melhor $\mathrm{QV}$, foram obtidas nos domínios: Função Social, Dirigir Automóvel e Visão de Cores, enquanto as mais baixas estiveram relacionadas à Saúde Geral e Visão Geral. Outros estudos também reportam escores mais elevados destes domínios em pacientes portadores de diferentes afecções oftálmi$\operatorname{cas}^{20,25} \mathrm{e}$ em indivíduos saudáveis ${ }^{26}$.

No presente estudo, a pior QVRFV no domínio Saúde Geral, pode ser atribuída à condição clínica dos idosos, relacionada às comorbidades, à polifarmácia e outras variáveis não identificadas na caracterização da amostra. Com respeito à pontuação na subescala Visão Geral, pode-se associá-la à baixa AV (para longe e/ou para perto) apresentada pela maioria dos idosos. Por outro lado, a pontuação elevada no domínio Dirigir Automóvel deve ser interpretada com cautela. Considerando o número elevado de itens perdidos (missings) neste domínio, pode-se apreender que dentre os 100 idosos entrevistados somente os 10 que responderam às questões deste grupo dirigiam automóvel e não percebiam dificuldade, ou prejuízo, nesta atividade pela queda da AV.

A análise de regressão univariada apontou as variáveis QVRFV e AV (esta com significância estatística limítrofe) como preditoras independentes da adesão, ratificando a suposição central das análises efetuadas neste estudo.
Em relação à QVRFV, possivelmente os sujeitos que avaliaram esse construto de forma mais positiva tinham a percepção de maior autonomia e autoeficácia, decorrentes de um repertório individual mais rico de recursos e habilidades que permitiam maior independência e segurança no manejo das medicações em uso, o que poderia contribuir para maior adesão.

De forma semelhante, a AV também se mostrou preditora da adesão. É possível inferir que a baixa AV interfere de forma negativa na operacionalização do uso dos medicamentos, implicando em dificuldades diversas que podem estar relacionadas desde o reconhecimento do rótulo, das cartelas, das cápsulas/comprimidos, até o preparo/estabelecimento da dosagem adequada, e ainda nas formas de aquisição dos medicamentos.

Considerando que tanto a qualidade de vida como a AV foram preditoras da adesão e que a avaliação da qualidade de vida estava relacionada à função visual, seria possível supor a AV como variável moderadora da relação entre QVRFV e adesão. Assim, nos sujeitos com pior AV (e, portanto, com menores recursos individuais para o manejo das medicações) seria percebida pior QVRFV, a qual mostraria correlação positiva com a não adesão. $O$ mesmo não ocorreria para os sujeitos com AV normal.

Para verificar essa suposição, deu-se seqüência à análise do efeito moderador da AV na relação entre QVRFV e adesão medicamentosa. Um moderador é uma variável qualitativa (neste estudo, AV normal ou rebaixada) ou quantitativa que afeta a direção e/ou a força da relação entre uma variável independente, ou preditora, e uma variável dependente, ou critério. Do ponto de vista correlacional, um moderador é uma terceira variável que afeta a correlação de ordem-zero entre duas outras variáveis, modificando a direção da correlação ou reduzindo substancialmente a magnitude desta correlaçãa ${ }^{18}$.

$\mathrm{Na}$ análise do efeito moderador, entretanto, observou-se que a AV não modera a relação entre QVRFV e (não)adesão, ratificando que o efeito de ambas sobre a adesão ocorre de forma independente.

\section{Conclusão}

A presente investigação é inédita na indicação de que a QVRFV é um fator preditor da adesão medicamentosa em idosos, ou seja, quanto pior a percepção de qualidade de vida relacionada à visão, menor a adesão.

A ausência do efeito moderador da $A V$ significa que independente da relação desta variável com a QVRFV, esta última é, por si só, um importante determinante da adesão, ou seja, a percepção sobre o impacto da visão na qualidade de vida interfere diretamente na adesão ao uso dos medicamentos. Embora a análise comparativa entre a AV e a pontuação do NEI VFQ-25 tenha mostrado relação positiva entre estas variáveis, entende-se que a percepção de piora da QVRFV não pode ser atribuída ap- 
enas à $\mathrm{AV}$, mas a outros fatores intrínsecos e extrínsecos ao sujeito, não mensurados neste estudo. Em outras palavras, a relação entre QVRFV e adesão medicamentosa não é afetada pela $\mathrm{AV}$, mas possivelmente pelo conjunto de facetas que compõem esse constructo.

Para os idosos com RD, cujo prognóstico visual nem sempre é favorável à manutenção da visão normal ou próxima do normal, a QVRFV torna-se relevante nesse prognóstico, dada a sua relação com a adesão para o controle clínico do DM e da HAS. Somam-se a isso as limitações comumente manifestas na velhice, que podem contribuir de forma negativa na QVRFV e, por conseguinte, na adesão ao uso dos medicamentos.

Portanto, para a prática educativa do enfermeiro ressalta-se a importância de intervenções que visem aperfeiçoar a adesão entre os idosos com baixa AV e entre aqueles que revelam pior QVRFV, ainda que não apresentem queda acentuada da AV.

\section{REFERÊNCIAS}

1.Fowler MJ. Microvascular and macrovascular complications of Diabetes.Clin Diabetes. 2008;26(2):77-82.

2.Sociedade Brasileira de Diabetes. Diretrizes da Sociedade Brasileira de Diabetes: 2013-2014. São Paulo: AC Farmacêutica; 2014.

3.Jongsareejit A, Potisat S, Krairittichai U, Sattaputh C, Arunratanachote W.The Thai DMS diabetes complications (DD.Comp.) project: prevalence and risk factors of diabetic retinopathy in Thai patients with type 2 diabetes mellitus.J Med Assoc Thai. 2013; 96: 1476-82.

4.Hammes HP.Optimal treatment of diabetic retinopathy. TherAdvEndocrinolMetab. 2013;4 (2):61-71.

5.The UK Prospective Study Group. Tight blood pressure control and risk of macrovascular and microvascular complications in patients with type 2 diabetes (UKPDS). BMJ. 1998;317: 703-12.

6.Gross JL, Nehme M. Detecção e tratamento das complicações crônicas do diabetes melito: Consenso da Sociedade Brasileira de Diabetes e Conselho Brasileiro de Oftalmologia.RevAssocMed Bras.1999;45: 279-84.

7.Andrade NHS, Zanetti ML, Santos MA. A percepção visual de pacientes com retinopatia diabética, segundo o referencial de Merleau-Ponty. Rev enferm UERJ. 2008;16: 249-54.

8.Baron RM, Kenny DA. The moderator-mediator variable distinction in social psychological research: conceptual, strategic, and statistical consideration. J PersSocPsychol. 1986;51:1173-82.

9.Leite SN, Vasconcellos MPC. Adesão à terapêutica medicamentosa: elementos para a discussão de conceitos e pressupostos adotados na literatura.Ciênc Saúde Coletiva. 2003;8: 775-82.

10.Balkrishnan R, Rajagopalan R, Camacho FT, Huston AS, Murray FT, Anderson RT. Predictors of medication adherence and associated health care cost in an older population with type 2 Diabetes Mellitus: a longitudinal cohort study. ClinTher. 2003;25:2958-71.

11.Hughes CM. Medication non-adherence in the el- derly. Drugs Aging. 2004;21:793-811.

12.Murray MD, Morrow DG, Weiner M, Clark DD, Tu W, Deer MM et al. A conceptual framework to study medication adherence in older adults.Am J GeriatrPharmacother.2004;2:36-43.

13.Cintra FA, Guariento ME, Miyazaki LA. Adesão Medicamentosa em idosos em seguimento ambulatorial. Ciênc Saúde Coletiva. 2010;15(suppl3):3507-15.

14. Tabachnick BG, Fidell LS. Using Multivariate Statistics. 4th ed. Boston: Allyn and Bacon; 2001.

15.Chatkin JM, Cavalet-Blanco D, Scaglia NC, Tonietto RG, Wagner MB, Fritscher, CC. Adesão ao tratamento de manutenção em asma (estudo ADERE). J BrasPneumol. 2006;32:277-83.

16. World Health Organization (WHO). CDC. Centers for Disease Control and Prevention.Classifications of Diseases, Functioning, and Disability [online].International Classification of Diseases, Tenth Revision, Clinical Modification (ICD-10-CM).ICD-10-CM Files via FTP: 2014. Dtab12.zip. ICD-10-CM Tabular list of diseases and injuries (FY12).H54.7. Unspecified visual loss.p.356. [cited in 2014 Jan 23] Available from: URL:http://www. cdc.gov/nchs/icd/icd $10 \mathrm{~cm}$. htm

17.Javitt J, Brauweiler HP, Jacobi KW, Klemen U, Kohner S. Cataract extraction with multifocal intraocular lens implantation: clinical, functional, and quality-of-life outcomes. Multicenter clinical trial in Germany and Austria.J Cataract Refract Surg. 2000; 26: 1356-66.

18.Katina JH, Lyra JMAG, Souza CD, Trindade FC. Tratamento cirúrgico da catarata pediátrica. Arq Bras Oftalmol. 2002; 65: 193-7.

19.Mangione CM, Lee PP, Gutierrez PR, Spritzer K, Berry $\mathrm{S}$, Hays RD. Development of the 25-Item National Eye Institute Visual Function Questionnaire. Arch Ophthalmol. 2001; 119: 1050-8.

20.Fonseca ICM. Adaptação cultural do National EyeInstitute Visual Functioning Questionnaire (NEI VFQ-25) para idosos brasileiros com baixa visão [dissertação de mestrado]. Campinas (SP): Universidade Estadual de Campinas; 2006. 21.Hashmi SK, Afridi MB, Abbas K, Sajwani RA, Saleheen D, Frossard PM et al. Factors associated with adherence to anti-hypertensive treatment in Parkinson. PloS ONE.2007;2(3): e280.

22.Ventura-Cerdá JM, Mínguez-Gallago C, FernándezVellalba EM, Alós-Almiñana M, Andrés-Soler J. Escalasimplificadaparadetectarproblemas de adherencia (ESPA) al tratamientoantirretroviral. Farm Hosp. 2006;30(3):171-6. 23.Delgado AB, Lima ML. Contributo para a validação concorrente de uma medida de adesão aos tratamentos. PsicSaúde\&Doenças. 2001;2 (2):81-100.

24.Chew EY, Klein ML, Ferris III FL, Remaley NA, Murphy RP, Chantry K et al. Association of elevated serum lipid levels with retinal hard exudates in diabetic retinopathy: Early Treatment Diabetic Retinopathy Study (ETDRS) Repot 22. Arch Ophthalmol. 1996;114: 1079-84. 25.Hoeksema L, Los LI.Vision-related quality of life in herpetic anterior uveitis patients. PLoS One. 2014;9 (1):e85224. 26.Globe D, Varma R, Azen SP, Paz S, Yu E, Peston-Martin $S$ et al. Psychometric performance of the NEI VFQ-25 in visually normal Latinos: The Los Angeles Latino Eye Study. Invest Ophthalmol Vis Sci. 2003;44: 1470-8. 